PROCEEDINGS OF THE

AMERICAN MATHEMATICAL SOCIETY

Volume 126, Number 2, February 1998, Pages 515-522

S $0002-9939(98) 04054-4$

\title{
CORRECTED OUTER FUNCTIONS
}

\author{
EVGUENI DOUBTSOV
}

(Communicated by Albert Baernstein II)

\begin{abstract}
Given $0<p<2$ and a strictly positive continuous function $\varphi$ on the unit circle, we construct a bounded analytic function $g$ such that $\left|g^{*}\right|=\varphi$ a.e., and $g$ is in the Besov space $A_{p}^{1}$ on the unit disc.
\end{abstract}

\section{INTRODUCTION}

Let $\mathbb{D}=\{z \in \mathbb{C}:|z|<1\}, \mathbb{T}=\{\zeta \in \mathbb{C}:|\zeta|=1\}$ be the unit disc and the unit circle. Denote by $m_{2}$ and $m_{1}$ the corresponding Lebesgue measures, $m_{2}(\mathbb{D})=1$, $m_{1}(\mathbb{T})=1 . H(\mathbb{D})$ is the space of all analytic functions $f: \mathbb{D} \rightarrow \mathbb{C}$.

Put $H^{\infty}:=\{f \in H(\mathbb{D}): f$ is bounded $\}, A(\mathbb{D})=H(\mathbb{D}) \cap C(\overline{\mathbb{D}})$ (the disc-algebra). Let $0<p \leq 2$; then define

$$
\begin{aligned}
A_{p}^{1}(\mathbb{D})=\left\{f \in H(\mathbb{D}):\|f\|_{A_{p}^{1}(\mathbb{D})}^{p}\right. & =\left\|f^{\prime}\right\|_{A_{p}(\mathbb{D})}^{p} \\
& \left.=\int_{\mathbb{D}}\left|f^{\prime}(z)\right|^{p}(1-|z|)^{p-1} d m_{2}(z)<\infty\right\} .
\end{aligned}
$$

Since $\|\cdot\|_{A_{p}^{1}(\mathbb{D})}$ is the Besov (quasi) norm, we say that $A_{p}^{1}(\mathbb{D})$ is the analytic Besov space. Recall some inclusions between $A_{p}^{1}(\mathbb{D})$ and other classical spaces of analytic functions. Let $\ell_{A}^{p}=\left\{f \in H(\mathbb{D}):\{\hat{f}(n)\}_{n \geq 0} \in \ell^{p}\right\}$ and $H^{p}$ be the Hardy class; then $\ell_{A}^{p} \subset A_{p}^{1}(\mathbb{D}) \subset H^{p}, 0<p \leq 2$. In particular, $A_{2}^{1}(\mathbb{D})=H^{2}$.

The aim of the present paper is to prove the following result (we use the symbol $g^{*}$ to denote the boundary values of $\left.g \in H^{\infty}\right)$.

Theorem. Let $0<p<2$ and $\varphi \in C(\mathbb{T}), \varphi>0$. Then there exists a function $g \in H^{\infty} \cap A_{p}^{1}(\mathbb{D})$ such that $\left|g^{*}\right|=\varphi m_{1}$-almost everywhere.

Remark. This theorem holds also for some non-continuous functions $\varphi$ and for nonnegative $\varphi$ with some zeros. Moreover, these results are true in the unit ball of $\mathbb{C}^{n}$, $n \geq 2$. We will not discuss these generalizations in the present paper.

If $p=1$, then the result under question was obtained in [2]. Note that the theorem is interesting for small $p>0$, since $H^{\infty} \cap A_{p}^{1}(\mathbb{D}) \subset H^{\infty} \cap A_{q}^{1}(\mathbb{D})$ if $0<p<q$.

Received by the editors March 13, 1996 and, in revised form, August 13, 1996.

1991 Mathematics Subject Classification. Primary 30D50; Secondary 30D55.

Key words and phrases. Hardy classes, Besov space, inner-outer factorization.

Supported by the Centre de Recerca Matemàtica, Institut d'Estudis Catalans (Barcelona) under a grant from DGICYT (Spain).

(C)1998 American Mathematical Society 
Indeed, suppose that $f \in H^{\infty}$; then, by Cauchy's inequality, $\left|f^{\prime}(z)\right|(1-|z|) \leq$ const, $z \in \mathbb{D}$, and therefore

$$
\int_{\mathbb{D}}\left|f^{\prime}(z)\right|^{(q-p)+p}(1-|z|)^{(q-p)+p-1} d m_{2}(z) \leq \mathrm{const} \int_{\mathbb{D}}\left|f^{\prime}(z)\right|^{p}(1-|z|)^{p-1} d m_{2}(z) .
$$

To prove the theorem, we apply, as in [2], the approximation construction of A. B. Aleksandrov in $L^{p}(\mathbb{T}), 0<p<1$ (see [1]). Recall that in [1] this construction yields a solution of the inner function problem in the unit ball of $\mathbb{C}^{n}$.

\section{Comments.}

1. The point of the theorem is the restriction $g \in A_{p}^{1}(\mathbb{D})$. Indeed, given a bounded modulus $\varphi \geq 0, \log \varphi \in L^{1}(\mathbb{T})$, the classical outer (in sense of Beurling) function is defined by the formula

$$
O_{\varphi}(z)=\exp \left(\int_{\mathbb{T}} \frac{\zeta+z}{\zeta-z} \log \varphi(\zeta) d m_{1}(\zeta)\right), \quad z \in \mathbb{D} .
$$

Recall that $O_{\varphi}$ satisfies the equality under consideration $\left|O_{\varphi}^{*}\right|=\varphi m_{1}$-a.e. (for further details about the inner-outer factorization see [3]). Therefore, it is important to note that there exists $\varphi \in C(\mathbb{T}), \varphi>0$, such that $O_{\varphi} \notin A_{p}^{1}(\mathbb{D})$ for all $0<p<2$ (this has been known for a long time, at least for $p=1$, see [5] and [6]). For example, the following argument gives the proof:

If $f \in A_{p}^{1}(\mathbb{D}), 1 \leq p<2$, then $\left\{\hat{f}\left(2^{n}\right)\right\}_{n \geq 0} \in \ell^{p}$. On the other hand, given a sequence $\left\{x_{n}\right\}_{n>0} \in \ell^{2}$, there exists $g \in A(\mathbb{D})$ such that $\hat{g}\left(2^{n}\right)=x_{n}, n \in \mathbb{Z}_{+}$. Hence, if we take $\left\{x_{n}\right\} \in \ell^{2} \backslash \ell^{p}$ for all $0<p<2$, we obtain a function $g \in A(\mathbb{D}) \backslash A_{p}^{1}(\mathbb{D})$ for all $0<p<2$. To finish the argument, put $h=g+2\|g\|_{\infty}$; then $|h|>0$ and $h$ is outer.

2. The theorem has an interpretation in terms of the inner-outer factorization. Indeed, let $0<q<2, \varphi \in C(\mathbb{T}), \varphi>0$, and $O_{\varphi} \notin \bigcup_{0<p<2} A_{p}^{1}(\mathbb{D})$. Then there exists an inner function $I_{\varphi}$ such that $O_{\varphi} I_{\varphi} \in A_{q}^{1}(\mathbb{D})$. In other words, the outer function $O_{\varphi}$ is corrected by $I_{\varphi}$.

Given a space $\mathcal{E} \subset H(\mathbb{D})$, recall one notion which is important for investigation of $z$-invariant subspaces of $\mathcal{E}$.

Definition. Let $I$ be an inner function. We say that $I$ divides $\mathcal{E}$ if

$$
I F \in \mathcal{E} \Longrightarrow F \in \mathcal{E} \quad \text { for all } F \in H^{q}, q>0 .
$$

Let $\varphi$ be as above; then the theorem says, in particular, that $I_{\varphi}$ does not divide $H^{\infty} \cap A_{p}^{1}(\mathbb{D})$. We refer the reader to the paper [8] for other results on division and non-division by inner functions in the spaces $H^{\infty} \cap A_{p}^{1}(\mathbb{D}), 0<p<2$.

3. Let $0<p, q<\infty$. Then define

$$
A_{p q}^{1}(\mathbb{D})=\left\{f \in H(\mathbb{D}): \int_{\mathbb{D}}\left|f^{\prime}(z)\right|^{p}(1-|z|)^{q-1} d m_{2}(z)<\infty\right\} .
$$

It is necessary to explain why we consider the case $q=p$ only.

Indeed, let $q>p$ and $f \in H^{\infty}$; then

$$
\int_{\mathbb{D}}\left|f^{\prime}(z)\right|^{p}(1-|z|)^{q-1} d m_{2}(z) \leq \mathrm{const} \int_{\mathbb{D}}(1-|z|)^{q-p-1} d m_{2}(z)<\infty,
$$


in other words, $H^{\infty} \subset A_{p q}^{1}(\mathbb{D})$, and the theorem is trivial in this case.

On the other hand, let $p>q>p-1, q \geq 1$ and $f \in A_{p q}^{1}(\mathbb{D})$; then (see, e.g, [4, p.67])

$$
\int_{-\pi}^{\pi}|t|^{q-p-1} \int_{-\pi}^{\pi}\left|f\left(e^{i(x+t)}\right)-f\left(e^{i x}\right)\right|^{p} d x d t<\infty .
$$

Since there exists a modulus $\varphi \in C(\mathbb{T}), \varphi>0$, such that

$$
\int_{-\pi}^{\pi}|t|^{q-p-1} \int_{-\pi}^{\pi}\left|\varphi\left(e^{i(x+t)}\right)-\varphi\left(e^{i x}\right)\right|^{p} d x d t=\infty,
$$

the theorem is not valid for all $q<p$.

\section{Auxiliary Results}

Lemma 2.1 (see, e.g., [7, p.17], where the proof is given even in the ball of $\mathbb{C}^{n}$ ). Let $w \in \mathbb{D}, a>0, b>-1$. Then

$$
\begin{aligned}
\int_{\mathbb{T}} \frac{d m_{1}(\zeta)}{|1-\zeta \bar{w}|^{1+a}} & \leq \frac{\operatorname{const}(a)}{(1-|w|)^{a}}, \\
\int_{\mathbb{D}} \frac{(1-|z|)^{b} d m_{2}(z)}{|1-z \bar{w}|^{2+a+b}} & \leq \frac{\operatorname{const}(a, b)}{(1-|w|)^{a}} .
\end{aligned}
$$

Lemma 2.2. Let $d \in \mathbb{N}, 0<p<1, p d \geq 2$, and

$$
h(t, z)=\frac{i}{(2+t-t z)^{d}}, \quad t \geq 2, z \in \overline{\mathbb{D}} .
$$

Then there exist constants $\alpha=\alpha(p, d) \in(0,1)$ and $M_{0}=M_{0}(p, d) \geq 4$ such that

$$
\begin{gathered}
\frac{1}{2 \pi} \int_{-\pi}^{\pi}\left|\mathbb{1}_{[-\Delta, \Delta]}(\theta)-\operatorname{Re} h\left(M_{0} \Delta^{-1}, e^{i \theta}\right)\right|^{p} d \theta<\alpha \cdot \Delta / \pi, \\
\left\|h\left(M_{0} \Delta^{-1}, \cdot\right)\right\|_{L^{p}(\mathbb{T})}^{p}<\Delta / \pi \\
\left\|h^{\prime}\left(M_{0} \Delta^{-1}, \cdot\right)\right\|_{A_{p}(\mathbb{D})}^{p}<\Delta / \pi
\end{gathered}
$$

for all $\Delta \in(0, \pi / 4)$.

Proof. 1. To prove (2.1), we estimate the value of

$$
\operatorname{Re} h\left(t, e^{i \theta}\right)=\frac{-\operatorname{Im}(2+t(1-\cos \theta)+i t \sin \theta)^{d}}{|2+t(1-\cos \theta)-i t \sin \theta|^{2 d}} .
$$

If $|t \theta|<1$, then $|2+t(1-\cos \theta)+i t \sin \theta| \leq 4$. On the other hand, there exists $\varepsilon=\varepsilon(d) \in(0,1)$ such that

$$
2\left|\operatorname{Im}(2+t(1-\cos \theta)+i t \sin \theta)^{d}\right| \geq 2^{d-1}|t \theta| \quad \text { for all }|t \theta|<\varepsilon, t \geq 2
$$

(we killed the higher degrees of $|t \theta|$ ). Put $t=M \Delta^{-1}, M \geq 2$; then

$$
\frac{1}{2 \pi} \int_{-\Delta}^{\Delta}\left|\operatorname{Re} h\left(t, e^{i \theta}\right)\right|^{2} d \theta>\frac{1}{2 \pi} \int_{-\varepsilon / t}^{\varepsilon / t}\left|\operatorname{Re} h\left(t, e^{i \theta}\right)\right|^{2} d \theta \geq C(d) t^{-1}=C(d) \frac{\Delta}{M} .
$$

Let $0<p<1$ and $x \in[-1,1]$; then

$$
\frac{1}{2}\left((1+x)^{p}+(1-x)^{p}\right) \leq 1-\frac{p(1-p)}{2} x^{2} .
$$


Note that $\operatorname{Re} h\left(t, e^{-i \theta}\right)=-\operatorname{Re} h\left(t, e^{i \theta}\right) ;$ thus

$$
\frac{1}{2 \pi} \int_{-\Delta}^{\Delta}\left|1-\operatorname{Re} h\left(t, e^{i \theta}\right)\right|^{p} d \theta<\Delta\left(\frac{1}{\pi}-\frac{C_{1}(p, d)}{M}\right),
$$

where $C_{1}(p, d)>0$.

Now we estimate the complementary integral.

If $|\theta| \in[\Delta, \pi / 2]$, then $\left|h\left(t, e^{i \theta}\right)\right| \leq|t \sin \theta|^{-d} \leq C(d)|t \theta|^{-d}$. On the other hand, if $|\theta| \in[\pi / 2, \pi]$, then $\left|h\left(t, e^{i \theta}\right)\right| \leq|t|^{-d} \leq C(d)|t \theta|^{-d}$. For $t=M \Delta^{-1}$ we obtain

$$
\begin{aligned}
\frac{1}{2 \pi} \int_{[-\pi, \pi] \backslash[-\Delta, \Delta]}\left|\operatorname{Re} h\left(t, e^{i \theta}\right)\right|^{p} d \theta & \leq C(d) \int_{\Delta}^{\infty}(t \theta)^{-p d} d \theta \\
& \leq \Delta \frac{M^{1-p d} C_{2}(p, d)}{M} .
\end{aligned}
$$

If $M_{1}$ is so large that $C_{1}(p, d)>M_{1}^{1-p d} C_{2}(p, d)$ and $M_{0} \geq M_{1}$, then (2.1) follows from (2.4) and (2.5).

2. Lemma 2.1, with $a=p d-1>0$, yields

$$
\begin{aligned}
\|h(t, \zeta)\|_{L^{p}(\mathbb{T})}^{p} & =\int_{\mathbb{T}} \frac{d m_{1}(\zeta)}{|2+t-t \zeta|^{p d}} \\
& =\int_{\mathbb{T}} \frac{(t+2)^{-p d}}{\left|1-t(t+2)^{-1} \zeta\right|^{p d}} d m_{1}(\zeta) \\
& \leq \operatorname{const}(p, d) \frac{(t+2)^{-p d}}{\left(1-t(t+2)^{-1}\right)^{p d-1}} \\
& \leq \operatorname{const}_{2}(p, d) t^{-1} .
\end{aligned}
$$

Let $t^{-1}=\Delta M_{2}^{-1}$ and $M_{2}>\pi \cdot \operatorname{const}_{2}(p, d)$; then (2.2) holds if $M_{0} \geq M_{2}$.

3. We have

$$
\left|h^{\prime}(t, z)\right|=\frac{t d}{|2+t-t z|^{d+1}} .
$$

Hence, by Lemma 2.1, with $a=p d-1>0$ and $b=p-1>-1$, we obtain

$$
\begin{aligned}
\left\|h^{\prime}(t, z)\right\|_{A_{p}(\mathbb{D})}^{p} & =\int_{\mathbb{D}} \frac{t^{p} d^{p}(t+2)^{-p d-p}(1-|z|)^{p-1}}{\left|1-t(t+2)^{-1} z\right|^{p d+p}} d m_{2}(z) \\
& \leq \operatorname{const}(p, d) \frac{(t+2)^{-p d}}{\left(1-t(t+2)^{-1}\right)^{p d-1}} \\
& \leq \operatorname{const}_{3}(p, d) t^{-1} .
\end{aligned}
$$

Again, let $t^{-1}=\Delta M_{3}^{-1}$ and $M_{3}>\pi \cdot \operatorname{const}_{3}(p, d)$; then (2.3) holds if $M_{0} \geq M_{3}$. To finish the proof, define $M_{0}=\max \left\{4, M_{1}, M_{2}, M_{3}\right\}$.

\section{Elementary functions}

Given $R>0$, define $\mathbb{D}(R)=\{z \in \mathbb{C}:|z|<R\}$.

Lemma 3.1. Suppose that $p \in(0,1)$. Then there exists a constant $\beta=\beta(p) \in$ $(0,1)$ with the following property: Let $r \in(0,1 / 4)$ and $Q=\left(e^{-3 r i}, e^{3 r i}\right) \subset \mathbb{T}$. Let $\varkappa \in(0,1), R \in(0,1)$. Then there exists a function $f \in A(\mathbb{D})$ such that

$$
\begin{gathered}
\operatorname{Re} f<1 \text { on } Q, \quad \text { and } \quad \operatorname{Re} f<\varkappa \text { on } \mathbb{T} \backslash Q, \\
\left\|\mathbb{1}_{Q}-\operatorname{Re} f\right\|_{L^{p}(\mathbb{T})}^{p}<\beta m_{1}(Q),
\end{gathered}
$$




$$
\begin{gathered}
\|f\|_{L^{p}(\mathbb{T})}^{p}<m_{1}(Q), \\
\left\|f^{\prime}\right\|_{A_{p}(\mathbb{D})}^{p}<m_{1}(Q), \\
|f(z)|<\varkappa \quad \text { if } z \in \mathbb{D}(R) .
\end{gathered}
$$

Remark. We will use this lemma when $m_{1}(Q)$ and $\varkappa$ are small and $R$ is close to 1 .

Proof. Let $0<\delta<\varkappa \min \left\{r,(1-R)^{2}\right\}$ and $r \delta^{-1}=N \in \mathbb{N}$ (note that $N \varkappa>1$ ). Define $\zeta_{1}=e^{-r i}, \zeta_{j+1}=e^{2 \delta i} \zeta_{j}, 1 \leq j \leq N-1$; then $\left\{\zeta_{j}\right\}_{j=1}^{N} \subset\left[e^{-r i}, e^{r i}\right]$. Take $d=d(p) \in \mathbb{N}$ such that $p d \geq 2$ (in particular, $d \geq 2$ ) and let $\alpha$ and $M_{0}$ be those given by Lemma 2.2. We claim that the function

$$
f(z):=\sum_{j=1}^{N} h_{j}(z):=\sum_{j=1}^{N} \frac{i}{\left(2+M_{0} \delta^{-1}\left(1-z \bar{\zeta}_{j}\right)\right)^{d}}
$$

satisfies the conditions of the lemma.

1. Let $\zeta \in \mathbb{T}$; then

$$
\left|2+M_{0} \delta^{-1}\left(1-\zeta \bar{\zeta}_{j}\right)\right| \geq \min \left(2, M_{0} \delta^{-1}\left|1-\zeta \bar{\zeta}_{j}\right|\right)=\min \left(2, M_{0} \delta^{-1}\left|\zeta-\zeta_{j}\right|\right) .
$$

Now, assume that $\zeta \in Q$ and $\min \left\{j: \arg (\zeta) \leq \arg \left(\zeta_{j}\right)\right\}=k$; then $\left|\zeta_{k+l}-\zeta\right| \geq l \delta$, $l=1,2, \ldots, N-k$. Therefore

$$
\sum_{j=k}^{N}\left|h_{j}(\zeta)\right| \leq 2^{-d}+\sum_{l=1}^{\infty}\left(l M_{0}\right)^{-d}
$$

Analogously,

$$
\sum_{j=1}^{k-1}\left|h_{j}(\zeta)\right| \leq 2^{-d}+\sum_{l=1}^{\infty}\left(l M_{0}\right)^{-d}
$$

Hence, if $\zeta \in Q$, then

$$
\frac{1}{2}|f(\zeta)| \leq 2^{-d}+\sum_{l=1}^{\infty}\left(l M_{0}\right)^{-d}
$$

Since $M_{0} \geq 4$, the first part of (3.1) holds.

If $\zeta \in \mathbb{T} \backslash Q$, then $\left|1-\zeta \bar{\zeta}_{j}\right| \geq r=N \delta$ for all $1 \leq j \leq N$. Hence

$$
|f(\zeta)| \leq \sum_{j=1}^{N}\left|h_{j}(\zeta)\right| \leq N \cdot N^{-d}<\varkappa^{d-1} \leq \varkappa .
$$

2. The estimate (2.1) from Lemma 2.2 , for $\Delta=\delta$, gives

$$
\begin{aligned}
\left\|\mathbb{1}_{Q}-\operatorname{Re} f\right\|_{L^{p}(\mathbb{T})}^{p} & \leq m_{1}(Q)-N \delta / \pi+\sum_{j=1}^{N}\left\|\mathbb{1}_{\left[\zeta_{j} e^{-\delta i}, \zeta_{j} e^{\delta i}\right]}-\operatorname{Re} h_{j}\right\|_{L^{p}(\mathbb{T})}^{p} \\
& <m_{1}(Q)-(N \delta-\alpha(p) N \delta) / \pi=m_{1}(Q)-(1-\alpha(p)) \cdot r / \pi \\
& <\beta(p) m_{1}(Q) .
\end{aligned}
$$

3. Since $\left\|h_{j}\right\|_{L^{p}(\mathbb{T})}^{p}<\delta / \pi($ see $(2.2))$, we obtain

$$
\|f\|_{L^{p}(\mathbb{T})}^{p} \leq \sum_{j=1}^{N}\left\|h_{j}\right\|_{L^{p}(\mathbb{T})}^{p}<N \delta / \pi=r / \pi<m_{1}(Q) .
$$


4. The property (2.3) yields

$$
\left\|f^{\prime}\right\|_{A_{p}(\mathbb{D})}^{p} \leq \sum_{j=1}^{N}\left\|h_{j}^{\prime}\right\|_{A_{p}(\mathbb{D})}^{p}<N \delta / \pi<m_{1}(Q) .
$$

5. If $|z|<R$, then $\left|1-z \bar{\zeta}_{j}\right|^{2} \geq(1-R)^{2}$ for all $1 \leq j \leq N$. Thus, as above,

$$
|f(z)| \leq N \cdot \delta^{d}(1-R)^{-d} \leq N \delta^{d / 2} \varkappa^{d / 2}<\varkappa .
$$

The proof is complete.

\section{Approximation construction}

Lemma 4.1. Let $0<p<1$. Then there exists a constant $\gamma=\gamma(p) \in(0,1)$ with the following property: Suppose that $\psi \in C(\mathbb{T}), \psi>0, R \in[0,1), \varepsilon>0$; then there exists a function $F \in A(\mathbb{D})$ such that

$$
\begin{gathered}
\operatorname{Re} F<\psi \quad \text { on } \mathbb{T}, \\
\|\psi-\operatorname{Re} F\|_{L^{p}(\mathbb{T})}^{p}<\gamma\|\psi\|_{L^{p}(\mathbb{T})}^{p}, \\
\|F\|_{L^{p}(\mathbb{T})}^{p}<\|\psi\|_{L^{p}(\mathbb{T})}^{p}, \\
\left\|F^{\prime}\right\|_{A_{p}(\mathbb{D})}^{p}<\|\psi\|_{L^{p}(\mathbb{T})}^{p}, \\
|F|<\varepsilon \quad \text { on } \quad \mathbb{D}(R) .
\end{gathered}
$$

Proof. Take a linear combination of characteristic functions $h:=\sum_{j=1}^{J} c_{j} \mathbb{1}_{Q_{j}}$ (the $\operatorname{arcs} Q_{j}$ are mutually disjoint and small enough, $c_{j}>0$ ) such that

$$
\begin{gathered}
2\|\psi-h\|_{L^{p}(\mathbb{T})}^{p}<(1-\beta(p))\|\psi\|_{L^{p}(\mathbb{T})}^{p}, \\
\psi-h \geq \eta \quad \text { for some } \eta>0 .
\end{gathered}
$$

Put $c_{0}=\max \left\{c_{j}: 1 \leq j \leq J\right\}$ and $\varkappa=\min \{\varepsilon, \eta\} /\left(2 c_{0} J\right)$. Given the $\operatorname{arcs} Q_{j}, \varkappa$ and $R$, Lemma 3.1 provides the functions $f_{j}$.

We claim that the function $F:=\sum_{j=1}^{J} c_{j} f_{j}$ satisfies the conditions of the lemma with $\gamma=(1+\beta) / 2$.

Since $2 c_{0} J \varkappa \leq \eta,(3.1)$ and (4.7) yield the inequality $\psi-\operatorname{Re} F \geq \eta / 2$, so (4.1) holds. By (3.2) and (4.6), we have (4.2). Indeed,

$$
\begin{aligned}
\|\psi-\operatorname{Re} F\|_{L^{p}(\mathbb{T})}^{p} & \leq\|\psi-h\|_{L^{p}(\mathbb{T})}^{p}+\sum_{j=1}^{J} c_{j}^{p}\left\|\mathbb{1}_{Q_{j}}-\operatorname{Re} f_{j}\right\|_{L^{p}(\mathbb{T})}^{p} \\
& <\frac{1}{2}(1-\beta)\|\psi\|_{L^{p}(\mathbb{T})}^{p}+\beta\|h\|_{L^{p}(\mathbb{T})}^{p} \\
& \leq \gamma\|\psi\|_{L^{p}(\mathbb{T})}^{p} .
\end{aligned}
$$

The property (3.3) provides the estimate

$$
\|F\|_{L^{p}(\mathbb{T})}^{p} \leq \sum_{j=1}^{J} c_{j}^{p}\left\|f_{j}\right\|_{L^{p}(\mathbb{T})}^{p}<\sum_{j=1}^{J} c_{j}^{p} m_{1}\left(Q_{j}\right) \leq\|\psi\|_{L^{p}(\mathbb{T})}^{p} .
$$

The same argument shows that (3.4) implies (4.4).

At last, $(3.5) \Rightarrow(4.5)$, since $2 c_{0} J \varkappa<\varepsilon$. 


\section{Proof of the THEOREM}

Proof. Given a strictly positive continuous modulus $\varphi$, put $\psi_{0}=\log \varphi$. Without loss of generality, we suppose that $\psi_{0}>0$ and $0<p<1$.

Put $\psi=\psi_{0}$ and $R=R_{0}:=0$; then Lemma 4.1 yields a function $F \in A(\mathbb{D})$. Define $F_{1}:=F$.

Suppose, as induction hypothesis, that $m \in \mathbb{N},\left\{F_{k}\right\}_{k=1}^{m} \subset A(\mathbb{D})$ and $\left\{R_{k}\right\}_{k=0}^{m-1} \subset$ $[0,1)$. Assume also that

$$
\begin{gathered}
\operatorname{Re}\left(\sum_{k=1}^{m} F_{k}\right)<\psi_{0} \quad \text { on } \quad \mathbb{T}, \\
\left\|\psi_{0}-\operatorname{Re}\left(\sum_{k=1}^{m} F_{k}\right)\right\|_{L^{p}(\mathbb{T})}^{p}<\gamma^{m}\left\|\psi_{0}\right\|_{L^{p}(\mathbb{T})}^{p}, \\
\left\|F_{m}\right\|_{L^{p}(\mathbb{T})}^{p}<\gamma^{m-1}\left\|\psi_{0}\right\|_{L^{p}(\mathbb{T})}^{p}, \\
\left\|F_{m}^{\prime}\right\|_{A_{p}(\mathbb{D})}^{p}<\gamma^{m-1}\left\|\psi_{0}\right\|_{L^{p}(\mathbb{T})}^{p}, \\
\left\|\sum_{k=1}^{m-1} F_{k}^{\prime}\right\|_{A_{p}\left(\mathbb{D} \backslash \mathbb{D}\left(R_{m-1}\right)\right)}^{p}<\gamma^{m-1}, \\
\left\|\left(\exp \left(F_{m}\right)-1\right)\left(\sum_{k=1}^{m-1} F_{k}^{\prime}\right)\right\|_{A_{p}\left(\mathbb{D}\left(R_{m-1}\right)\right)}^{p}<\gamma^{m-1} .
\end{gathered}
$$

Remark (base of induction). If $m=1$, then (5.1-5.4) are (4.1-4.4), and the estimates (5.5), (5.6) are trivial.

Step $m+1$. Define $R_{m}$ such that (5.5) holds for $\sum_{k=1}^{m}$ and $\gamma^{m}$. Take $\varepsilon_{m}>0$ such that

$$
\left\|\left(\exp \left(\varepsilon_{m}\right)-1\right)\left(\sum_{k=1}^{m} F_{k}^{\prime}\right)\right\|_{A_{p}\left(\mathbb{D}\left(R_{m}\right)\right)}^{p}<\gamma^{m} .
$$

Given $p \in(0,1)$ and $\psi=\psi_{0}-\operatorname{Re}\left(\sum_{k=1}^{m} F_{k}\right)>0, R=R_{m}, \varepsilon=\varepsilon_{m}$, Lemma 4.1 provides the function $F_{m+1}$.

Note that (4.1-4.4) $\Rightarrow(5.1-5.4)$ and (5.7) $\Rightarrow(5.6)$ for $m+1$. Now the induction construction proceeds.

Recall that $\sum_{m=1}^{\infty} \gamma^{m}<\infty$; therefore, by (5.3), the series $\sum_{k=1}^{\infty} F_{k}$ converges in $H^{p}$, so define

$$
g=\exp \left(\sum_{k=1}^{\infty} F_{k}\right)
$$

We claim that $g$ satisfies the conditions of the theorem.

Put $\Phi:=\max (\varphi)<+\infty$. Then (5.1) implies the estimate $|g| \leq \Phi$ on the disc $\mathbb{D}$, and therefore $g \in H^{\infty}$.

On the other hand, (5.2) yields the equality $\left|g^{*}\right|=\varphi m_{1}$-a.e. 
So we have to prove the property $g \in A_{p}^{1}(\mathbb{D})$ only. Introduce extra notations

$$
G_{m}=\left(\sum_{k=1}^{m} F_{k}^{\prime}\right) \exp \left(\sum_{k=1}^{m} F_{k}\right), \quad x_{m}=\left\|G_{m+1}-G_{m}\right\|_{A_{p}(\mathbb{D})}^{p} .
$$

It is sufficient to show that $\left\{x_{m}\right\}_{m=1}^{\infty} \in \ell^{1}$. Fix an integer $m \in \mathbb{N}$. Then

$$
\begin{aligned}
x_{m} \leq X_{m}+Y_{m}:= & \left\|F_{m+1}^{\prime} \exp \left(\sum_{k=1}^{m+1} F_{k}\right)\right\|_{A_{p}(\mathbb{D})}^{p} \\
& +\left\|\left[\sum_{k=1}^{m} F_{k}^{\prime}\right]\left[\exp \left(\sum_{k=1}^{m+1} F_{k}\right)-\exp \left(\sum_{k=1}^{m} F_{k}\right)\right]\right\|_{A_{p}(\mathbb{D})}^{p} .
\end{aligned}
$$

$\mathrm{X})$. The properties (5.1) and (5.4) imply the estimate

$$
X_{m} \leq \gamma^{m} \Phi\left\|\psi_{0}\right\|_{L^{p}(\mathbb{T})}^{p} .
$$

Y). By (5.1), (5.5) and (5.6), we obtain

$$
\begin{aligned}
Y_{m} & \leq 2 \Phi\left\|\sum_{k=1}^{m} F_{k}^{\prime}\right\|_{A_{p}\left(\mathbb{D} \backslash \mathbb{D}\left(R_{m}\right)\right)}^{p}+\Phi\left\|\left(\exp \left(F_{m+1}\right)-1\right)\left(\sum_{k=1}^{m} F_{k}^{\prime}\right)\right\|_{A_{p}\left(\mathbb{D}\left(R_{m}\right)\right)}^{p} \\
& \leq \gamma^{m} \cdot 3 \Phi .
\end{aligned}
$$

Since $\sum_{m=1}^{\infty} \gamma^{m}<\infty$, the items X) and Y) yield $\sum_{m=1}^{\infty} x_{m}<+\infty$.

The proof is finished.

\section{ACKNOWLEDGEMENTS}

The author is grateful to A. B. Aleksandrov for suggesting the problem considered, and to the referee for constructive remarks.

\section{REFERENCES}

1. A. B. Aleksandrov, The existence of inner functions in the ball, Mat. Sb. 118 (160), no. 2 (6) (1982), 147-163; English transl., Math. USSR-Sb. 46 (1983), 143-159. (Russian) MR 83i:32002

2. Y. Dupain, Gradients des fonctions intérieures dans la boule unité de $\mathbb{C}^{n}$, Math. Z. 193 (1986), 85-94. MR 87j:32006

3. J. Garnett, Bounded analytic functions, Academic Press, New York, 1981. MR 83g:30037

4. S. V. Hruscev and V. V. Peller, Hankel operators, best approximations, and stationary Gaussian processes, Uspekhi Mat. Nauk 37, no. 1 (1982), 53-124; English transl., Russian Math. Surveys 37 (1982), 61-144. (Russian)

5. S. N. Mergelyan, On one integral for analytic functions, Izvestiya Akad. Nauk SSSR 15 (1951), 395-400 (Russian). MR 13:335f

6. W. Rudin, The radial variation of analytic functions, Duke Math. J. 22 (1955), 235-242. MR $18: 27 \mathrm{~g}$

7. - Function Theory in the Unit Ball of $\mathbb{C}^{n}$, Grundlehren Math. Wiss., vol. 241, Springer, Berlin, 1980. MR 82i:32002

8. S. A. Vinogradov, Multiplication and division in the space of analytic functions with area integrable derivative, and in some related spaces, Zap. Nauchn. Sem. S. Petersburg. Otdel. Mat. Inst. Steklov. (POMI) 222 (1995), 45-77 (Russian); English transl. in J. Math. Sci.

Centre de Recerca Matemàtica, Institut d'Estudis Catalans, Apartat 50, E-08193 Bellaterra, Barcelona, Spain

E-mail address: doubtsov@crm.es

Current address: ul. Partizana Germana 14/117, kv. 335, 198205 St. Petersburg, Russia

E-mail address: es@dub.pdmi.ras.ru 